\title{
Mutagenicities and Endocrine-disrupting Activities of 1-Hydroxy-2-nitropyrene and 1-Hydroxy-5-nitropyrene
}

\author{
Takayuki Kameda,* Ayuko Akiyama, Morio Yoshita, Chihiro Tachikawa, Akira Toriba, \\ Ning Tang, and Kazuichi Hayakawa \\ Institute of Medical, Pharmaceutical and Health Sciences, Kanazawa University, Kakuma-machi, Kanazawa, Ishikawa 920-1192, \\ Japan
}

(Received April 8, 2011; Accepted April 18, 2011; Published online April 22, 2011)

The mutagenicities and endocrine-disrupting activities of two isomers of mononitrated 1-hydroxypyrene [1-hydroxy- $x$-nitropyrenes (1-OH- $x$-NPs); $x=2$ and 5], which are not only photoreaction products of 1-nitropyrene (1-NP) but also constituent of ambient airborne particles, were evaluated for the first time using the Ames plate incorporation assay and the yeast two-hybrid assay, respectively. The mutagenicity of 1-OH-5-NP was weakly positive in the absence of rat liver S9, but was enhanced up to 3-fold with the metabolic activation by S9. On the contrary, 1-OH-2-NP did not exhibit significant mutagenicity in the presence or absence of S9. 1-OH-5-NP showed weak estrogenic activity, but 1-OH-2-NP did not show any estrogenic activity. The concentration of 1-OH5 -NP that gave $10 \%$ of activity of $1.0 \times 10^{-6} \mathrm{M} 17 \beta$ estradiol $\left(E_{2}\right)$ was $5.4 \times 10^{-7}$ M. 1-OH-5-NP exhibited stronger antiestrogenic and antiandrogenic activities than 1-OH-2-NP. 1-OH-5-NP at a concentration of 1.0 $\times 10^{-6} \mathrm{M}$ inhibited 71 and $90 \%$ of $\beta$-galactosidase activity induced by $1.0 \times 10^{-9} \mathrm{M}$ of $\mathrm{E}_{2}$ and $1.0 \times 10^{-8} \mathrm{M}$ of $5 \alpha$-dihydrotestosterone (DHT), respectively. On the other hand, $1.0 \times 10^{-6} \mathrm{M}$ of 1-OH-2-NP inhibited 16 and $43 \%$ of $\beta$-galactosidase activity induced by $1.0 \times 10^{-9} \mathrm{M}$ of $\mathrm{E}_{2}$ and $1.0 \times 10^{-8} \mathrm{M}$ of DHT, respectively. These findings point out the need for determining the environmental sources and distribution of 1-OH-2-NP and 1-OH-5-NP as well as the other hydroxynitropyrene isomers.

Key words — nitropyrene, nitropyrenol, polycyclic aromatic hydrocarbon, mutagen, endocrine disruptor

*To whom correspondence should be addressed: Institute of Medical, Pharmaceutical and Health Sciences, Kanazawa University, Kakuma-machi, Kanazawa, Ishikawa 920-1192, Japan. Tel.: +81-76-234-4458; Fax: +81-76-234-4456; E-mail: kameda@p.kanazawa-u.ac.jp

\section{INTRODUCTION}

Numerous polycyclic aromatic hydrocarbons (PAHs) have been detected in organic extracts of airborne particles, and concerns are rising that they may affect human health through their mutagenic and carcinogenic effects. Nitrated polycyclic aromatic hydrocarbons (NPAHs) are also a class of mutagens/carcinogens found in the atmosphere, and some of them exhibit stronger mutagenicity/carcinogenicity than their parent PAHs. ${ }^{1)}$ 1-Nitropyrene (1-NP) is believed to be emitted into the atmosphere from combustion processes of fossil fuel such as diesel fuel ${ }^{2)}$ and is one of the most abundant NPAHs in the atmosphere. ${ }^{3)}$ 1-NP taken up by humans and animals is metabolized to hydroxynitropyrenes (OHNPs; Fig. 1), such as 1-hydroxy-3-nitropyrene (1-OH-3NP), 1-hydroxy-6-nitropyrene (1-OH-6-NP), and 1hydroxy-8-nitropyrene (1-OH-8-NP) in the presence of cytochrome P450 enzymes. ${ }^{4}{ }^{5)}$ We recently found that these OHNP isomers were also produced from a photoreaction of 1-NP in the atmosphere as well as the other isomers, 1-hydroxy-2-nitropyrene (1-OH-2-NP) and 1-hydroxy-5-nitropyrene (1-OH5-NP). ${ }^{6)}$ Several groups reported that $1-\mathrm{OH}-3-\mathrm{NP}$, 1-OH-6-NP, and 1-OH-8-NP are weakly muta-<smiles></smiles>

Fig. 1. Structure of OHNP 
genic, ${ }^{7-10)}$ but the mutagenicities of $1-\mathrm{OH}-2-\mathrm{NP}$ and 1-OH-5-NP are unknown.

Recently several kinds of PAH derivatives have been found to act as endocrine disruptors which may cause the dysfunction of human and wildlife endocrine systems, abnormal development of reproductive systems, and immunodeficiencies. For example, several monohydroxylated derivatives of PAHs (OHPAHs) have significant estrogenic/antiestrogenic activities, as shown by a reporter gene assay ${ }^{11)}$ and by a yeast two-hybrid assay system based on the ligand-dependent interaction of the estrogen receptor (ER) and its co-activator. ${ }^{12)}$ Furthermore, mono- and dihydroxy metabolites of PAHs appear to act as antiandrogenic chemicals, as shown by a reporter gene assay based on Chinese hamster ovary $(\mathrm{CHO})$ cells transiently cotransfected with a human androgen receptor (hAR) vector and an MMTV-LUC vector. ${ }^{13)}$ We have also found that 1-OH-3-NP, 1-OH-6-NP, and 1-OH-8-NP show significant estrogenic, antiestrogenic, and antiandrogenic activities in the yeast two-hybrid assay system. ${ }^{14)}$ These results imply that 1-OH-2-NP and 1-OH-5-NP, whose structures are similar to those of OHPAHs and other OHNP isomers, also exhibit endocrine-disrupting activities.

In this study, theirfore, we first examined the mutagenicities and endocrine-disrupting activities of 1-OH-2-NP and 1-OH-5-NP. For these analyses, we used the Ames plate incorporation assay and the yeast two-hybrid assay, respectively.

\section{MATERIALS AND METHODS}

Synthesis of OHNPs-1-OH-2-NP was synthesized by nitration of 1-hydroxypyrene (OHPy) by 4-nitro-4-methyl-2,3,5,6-tetrabromo2,5-cyclohexadien-1-one in diethyl ether at room temperature for $2 \mathrm{hr}$ according to the literatures. $^{6,15,16)}$ 1-OH-5-NP was obtained by a photoreaction of 1-NP according to the previous report. ${ }^{6}$ Each OHNP isomer was purified by preparative normal phase HPLC (SUPELCO, St. Louis, MO, U.S.A.; Supelcosil PLC-SI, $21.2 \mathrm{~mm}$ ID $\times 25 \mathrm{~mm}$, eluted with $\mathrm{CH}_{2} \mathrm{Cl}_{2}$ containing $0.5 \mathrm{mM} \mathrm{CH} \mathrm{CHOH}_{3} \mathrm{Co} 10 \mathrm{ml} / \mathrm{min}$ ). To identify the synthetic compounds, their EI-MS and ${ }^{1} \mathrm{H}$ NMR analyses were performed.

Chemicals — 4-Nitro-4-methyl-2,3,5,6-tetrabromo2,5-cyclohexadien-1-one was purchased from Tokyo Chemical Industry Co., Ltd (Tokyo, Japan).
1-NP and OHPy were obtained from Sigma-Aldrich (St. Louis, MO, U.S.A.). $17 \beta$-Estradiol $\left(\mathrm{E}_{2}\right)$ and $5 \alpha$-dihydrotestosterone (DHT) were purchased from Wako Pure Chemicals (Osaka, Japan). 4Hydroxytamoxifen (4-OHT) and hydroxyflutamide $(\mathrm{OHFl})$ were obtained from Sigma-Aldrich and Toronto Research Chemical Inc. (North York, Canada), respectively. Test compounds were dissolved in ethanol and stored at $-20^{\circ} \mathrm{C}$ until use. All other chemicals were of the highest quality available from commercial sources.

Mutagenicity Assay — Mutagenic activities of OHNPs were assayed with Salmonella typhimurium strains TA98 and TA100 according to the method developed by Maron and Ames ${ }^{17)}$ including a slight modification of preincubation ${ }^{18)}$ in the presence or absence of S9 mix.

Yeast Two-hybrid Assay — Estrogenic, antiestrogenic, androgenic, and antiandrogenic activities of OHNPs were evaluated with the yeast two-hybrid assay following Nishikawa's method with some modifications. ${ }^{12,19,20)}$ Briefly, yeast cells (Saccharomyces cerevisiae Y190) expressing human estrogen receptor $(\mathrm{hER} \alpha)$ and hAR or two-hybrid system control yeast cells (Saccharomyces cerevisiae Y190 transfected with the pGBK7-53 and pGADT7-T) were grown overnight at $30^{\circ} \mathrm{C}$ with shaking in synthetic defined medium free from tryptophan and leucine, and treated with each test compound at $30^{\circ} \mathrm{C}$ for $4 \mathrm{hr}$. After the incubation, the treated cells were collected and enzymatically digested with $1 \mathrm{mg} / \mathrm{ml}$ Zymolyase $20 \mathrm{~T}$ at $37^{\circ} \mathrm{C}$ for 30 min. 2-Nitrophenyl- $\beta$-D-galactoside was added to the lysate to a final concentration of $4 \mathrm{mg} / \mathrm{ml}$. After incubation at $30^{\circ} \mathrm{C}$ for $45 \mathrm{~min}$, the reaction was terminated by the addition of $1 \mathrm{M} \mathrm{Na}_{2} \mathrm{CO}_{3}$. The yeast debris was removed by centrifugation and the absorbance of supernatant was measured at $415 \mathrm{~nm}$. Estrogenic activity was evaluated by the $10 \%$ relative effective concentration $\left(\mathrm{REC}_{10}\right)$, which is defined as the concentration of the test compounds showing $10 \%$ of the highest $\beta$-galactosidase activity of $E_{2}$. Antiestrogenic and antiandrogenic activities were evaluated by $\mathrm{IC}_{20}$, which is the concentration of the test compounds that inhibit $20 \%$ of $\beta$ galactosidase activity induced by $1.0 \times 10^{-9} \mathrm{M} \mathrm{E}_{2}$ and $1.0 \times 10^{-8} \mathrm{M}$ DHT, respectively.

\section{RESULTS AND DISCUSSION}

Table 1 shows the mutagenicities of OHNPs ob- 
Table 1. Specific Mutagenicities of OHNPs and 1-NP, and Relative Mutagenicities of OHNPs to 1-NP Evaluated by Ames Assay

\begin{tabular}{|c|c|c|c|c|}
\hline \multirow[t]{2}{*}{ Compound } & \multicolumn{2}{|c|}{ TA98 } & \multicolumn{2}{|c|}{ TA100 } \\
\hline & $-\mathrm{S} 9$ & $+\mathrm{S} 9$ & $-\mathrm{S} 9$ & $+\mathrm{S} 9$ \\
\hline \multicolumn{5}{|c|}{ Specific mutagenicity $^{a)}$} \\
\hline 1-OH-2-NP & 28 & 46 & 118 & 164 \\
\hline 1-OH-5-NP & 73 & 255 & 209 & 589 \\
\hline $1-\mathrm{NP}$ & 1605 & 165 & 539 & 236 \\
\hline DMSO & 22 & 29 & 122 & 127 \\
\hline \multicolumn{5}{|c|}{ Relative mutagenicity to $\left.1-\mathrm{NP}^{b}\right)$} \\
\hline 1-OH-2-NP ${ }^{a)}$ & 0.02 & 0.3 & 0.2 & 0.7 \\
\hline $\left.1-\mathrm{OH}-5-\mathrm{NP}^{a}\right)$ & 0.05 & 1.5 & 0.4 & 2.5 \\
\hline 1-OH-3-NP & $0.3^{c)}$ & $0.6^{c)}$ & $1.4^{d)}$ & $0.8^{d)}$ \\
\hline 1-OH-6-NP & $0.06^{c)}$ & $1.5^{c)}$ & $2.5^{d)}$ & $2.4^{d)}$ \\
\hline 1-OH-8-NP & $0.09^{c)}$ & $0.3^{c)}$ & -e) $^{e}$ & $\underline{-}^{e)}$ \\
\hline
\end{tabular}

DMSO: used as a negative control. a) This study. Specific mutagenicity, expressed as revertants $/ 40$ nmol-test compound, was calculated by least squares linear regression from linear portion of doseresponse curve. b) Calculated based on the specific mutagenicities. c) Obtained from reference 9. d) Obtained from reference 10. e) Not available in reference 10.

Table 2. $\mathrm{REC}_{10}$ and $\mathrm{IC}_{20}$ Values for OHNPs and Reference Chemicals in Yeast Two-hybrid Assay

\begin{tabular}{|c|c|c|c|}
\hline \multirow[t]{2}{*}{ Compound } & \multirow{2}{*}{$\begin{array}{c}\text { REC }_{10}{ }^{c)} \\
\text { Estrogenic } \\
\text { activity }\end{array}$} & \multicolumn{2}{|c|}{$\mathrm{IC}_{20}{ }^{e)}$} \\
\hline & & $\begin{array}{c}\text { Antiestrogenic } \\
\text { activity }\end{array}$ & $\begin{array}{c}\text { Antiandrogenic } \\
\text { activity }\end{array}$ \\
\hline $1-\mathrm{OH}-2-\mathrm{NP}^{a)}$ & $-^{d)}$ & $1.3 \times 10^{-6}$ & $3.7 \times 10^{-7}$ \\
\hline $1-\mathrm{OH}-5-\mathrm{NP}^{a)}$ & $5.4 \times 10^{-7}$ & $2.5 \times 10^{-7}$ & $3.2 \times 10^{-8}$ \\
\hline $1-\mathrm{OH}-3-\mathrm{NP}^{b)}$ & $6.0 \times 10^{-7}$ & $1.1 \times 10^{-6}$ & $2.3 \times 10^{-7}$ \\
\hline $\left.1-\mathrm{OH}-6-\mathrm{NP}^{b}\right)$ & $6.0 \times 10^{-8}$ & $1.0 \times 10^{-6}$ & $3.1 \times 10^{-7}$ \\
\hline $1-\mathrm{OH}-8-\mathrm{NP}^{b)}$ & $9.0 \times 10^{-7}$ & $7.0 \times 10^{-7}$ & $5.1 \times 10^{-8}$ \\
\hline $\mathrm{E}_{2}^{a)}$ & $6.0 \times 10^{-11}$ & & \\
\hline $\left.4-\mathrm{OHT}^{a}\right)$ & & $5.3 \times 10^{-6}$ & \\
\hline $\mathrm{OHFl}^{a)}$ & & & $5.3 \times 10^{-6}$ \\
\hline
\end{tabular}

a) This study. b) Taken from reference $14 . \quad c$ ) Concentration of the test compounds showing $10 \%$ of the highest $\beta$-galactosidase activity of $\mathrm{E}_{2} . d$ ) Significant induction of $\beta$ galactosidase activity was not observed at concentrations between $1.0 \times 10^{-8}$ and $1.0 \times 10^{-6} \mathrm{M}$. $e$ ) Concentration of the test compounds that inhibit $20 \%$ of $\beta$-galactosidase activity induced by $10^{-9} \mathrm{M} \mathrm{E}_{2}$ or $10^{-8} \mathrm{M}$ DHT.

tained in this study. 1-OH-2-NP was not mutagenically active in either TA98 or TA100 with or without metabolic activation by S9. The mutagenicity of 1-OH-5-NP in the TA98 and TA100 strains was weakly positive in the absence of S9, i.e., the numbers of revertants were 2-3-fold greater than the number of spontaneous revertants in the negative control [dimethyl sulfoxide (DMSO)]. On the other hand, the mutagenic activities of 1-OH-5-NP in both the strains were enhanced approximately 3 -fold by the addition of S9. The mutagenicities of 1-OH-5NP relative to the mutagenicity of 1-NP are given in Table 1 together with those of the other OHNP isomers. With S9 activation, they were 1.5 and 2.5 in TA98 and TA100, respectively, i.e., the mutagenic- ity of 1-OH-5-NP was higher than that of 1-NP, as was the case of 1-OH-6-NP in the presence of S9. These results are consistent with previous findings that some OHNP isomers need metabolic activation to exhibit mutagenicity. $8,9,21)$

Figure 2 shows the estrogenic activities of $E_{2}$ and the two isomers of OHNP. $\beta$-Galactosidase activity increased with increasing $\mathrm{E}_{2}$ concentration, reaching a plateau at $1.0 \times 10^{-6} \mathrm{M}$. A significant induction of $\beta$-galactosidase activity was also observed for 1-OH-5-NP $\left(\mathrm{REC}_{10}=5.4 \times 10^{-7} \mathrm{M}\right)$, but no activity was observed for 1-OH-2-NP at concentrations between $1.0 \times 10^{-8}$ and $1.0 \times 10^{-6} \mathrm{M}$. The $\mathrm{REC}_{10}$ and $\mathrm{IC}_{20}$ values for the OHNP isomers obtained in this study and for the reference chemicals 


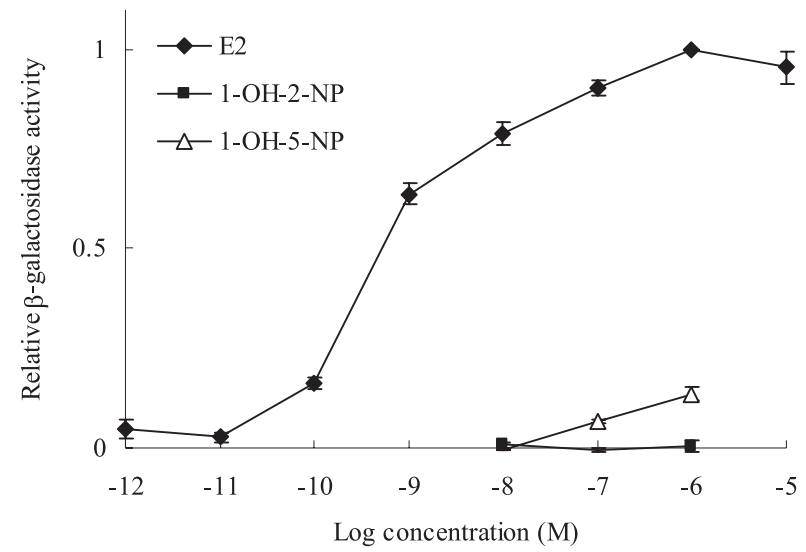

Fig. 2. Dose Response Curves of Estrogenic Activity of $E_{2}$ and 1-OH- $x$-NPs $(x=2$ and 5$)$ in a Yeast Two-hybrid Assay System

Each data point is the mean \pm S.D. $(n=3)$.

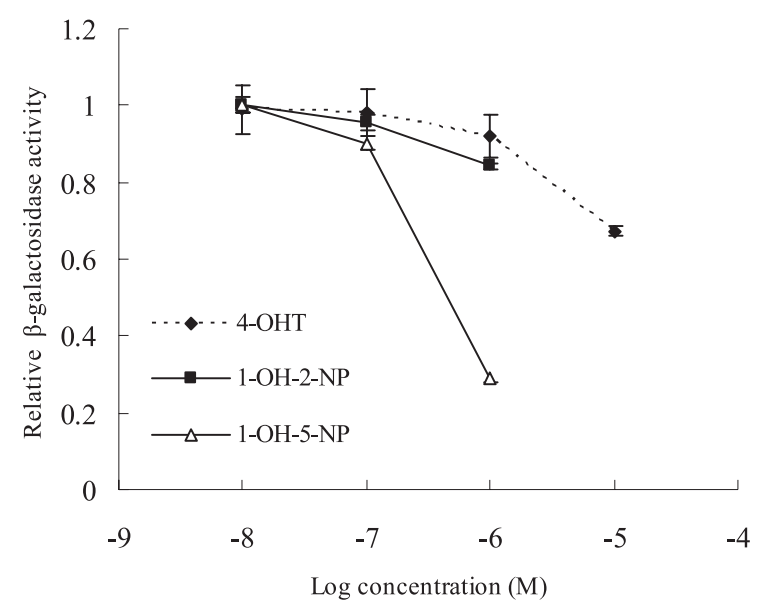

Fig. 3. Antiestrogenic Activity of 4-OHT and 1-OH- $x$-NPs ( $x=2$ and 5) against the Estrogenic Activity of $\mathrm{E}_{2}$ in a Yeast Two-hybrid Assay System

Antiestrogenic activities of 4-OHT and 1-OH- $x$-NPs were expressed as $\beta$-galactosidase activity relative to the level induced by $1.0 \times 10^{-9} \mathrm{M} \mathrm{E}_{2}$. Each data point is the mean \pm S.D. $(n=3)$.

are summarized in Table 2. The estrogenic activity of 1-OH-5-NP was lower than that of 1-OH-6-NP, but higher than the estrogenic activities of the other OHNP isomers previously reported ${ }^{14)}$ or bisphenol A $\left(\mathrm{REC}_{10}=3 \times 10^{-6} \mathrm{M}\right){ }^{12)}$ a known estrogenic compound. Figure 3 shows the antiestrogenic activities of the tested OHNPs in the concentration range from $1.0 \times 10^{-8}$ to $1.0 \times 10^{-6} \mathrm{M}$. To obtain these data, we used an $\mathrm{E}_{2}$ concentration of $1.0 \times$ $10^{-9} \mathrm{M}$, which induced about $50 \%$ of the maximum $\beta$-galactosidase activity. At a concentration of 1.0 $\times 10^{-6} \mathrm{M}$, each of the OHNP isomers decreased the induction of $\beta$-galactosidase activity by $\mathrm{E}_{2}$. $1-\mathrm{OH}-$ 2-NP and 1-OH-5-NP showed 4 and 21 times higher antiestrogenic activity, respectively, than 4-OHT, a typical ER antagonist (Table 2). Figure 4 shows

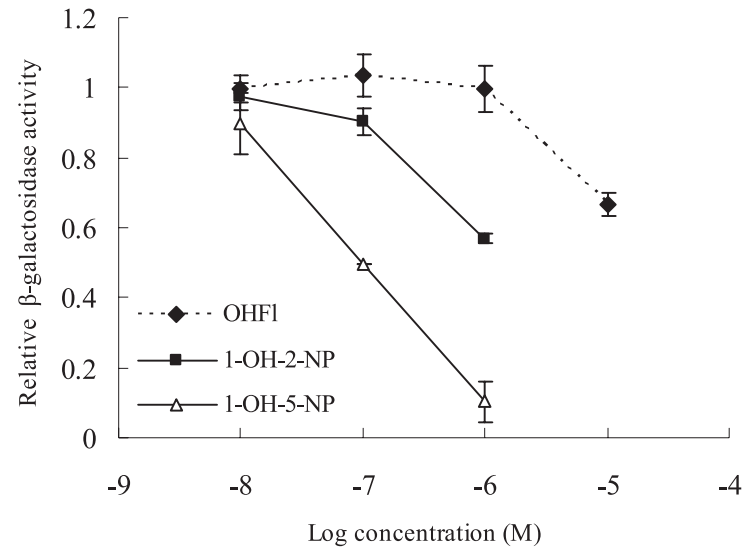

Fig. 4. Antiandrogenic Activity of OHFl and 1-OH- $x$-NPs ( $x=2$ and 5) against the Androgenic Activity of DHT in a Yeast Two-hybrid Assay System

Antiandrogenic activities of OHFl and $1-\mathrm{OH}-x$-NPs were expressed as $\beta$-galactosidase activity relative to the level induced by $1.0 \times 10^{-8} \mathrm{M}$ DHT. Each data point is the mean \pm S.D. $(n=3)$.

the results of the antiandrogenic activities for the tested OHNPs. In the presence of OHNPs at concentrations between $1.0 \times 10^{-8}$ and $1.0 \times 10^{-6} \mathrm{M}$, the activity of $1.0 \times 10^{-8} \mathrm{M}$ DHT, which induced about $50 \%$ of the highest $\beta$-galactosidase activity of DHT, was inhibited concentration-dependently. The highest inhibitory effect among the five OHNP isomers was observed with 1-OH-5-NP as was the case with antiestrogenic activity. The antiandrogenic activities of 1-OH-2-NP and 1-OH-5-NP were 14 and 166 times higher than the antiandrogenic activity of $\mathrm{OHFl}$ (Table 2). At concentrations less than $1.0 \times$ $10^{-6} \mathrm{M}$, neither OHNP isomer was cytotoxic to the control yeast cells, which supports the idea that the decreases of $\beta$-galactosidase induction observed in this study were due to antiestrogenic/antiandrogenic effects rather than cytotoxic effects. Neither 1-OH2-NP nor 1-OH-5-NP showed androgenic activity at concentrations between $1.0 \times 10^{-8}$ and $1.0 \times$ $10^{-6} \mathrm{M}$. OHPAHs having four aromatic rings, such as hydroxybenz $[a]$ anthracenes, hydroxychrysenes, and hydroxybenzo[c]phenanthrenes, were shown to have strong endocrine-disrupting activities. ${ }^{20)}$ In addition, it was reported that the four rings and a phenolic hydroxyl group needed to be in a rectangular plane in order for OHPAHs to bind to the site of the receptor. ${ }^{20)}$ The OHNP isomers have the same planar structure, which could account for their endocrine-disrupting activities.

Because of the significant biological effects of OHNPs, further studies of their environmental sources, sinks, and distributions are needed to better assess their risks. 
Acknowledgements This research was supported by MEXT/JSPS Grant-in-Aid for Scientific Research $(21200031,22510010,21390034)$, the Environment Research and Technology Development Fund (RF-0905) of the Ministry of the Environment, Japan, JSPS AA Scientific Platform Program, and Health and Labor Sciences Research Grants of Ministry of Health, Labor and Welfare, Japan. We thank Professor T. Nishihara, Osaka University, Japan for providing the yeast cells.

\section{REFERENCES}

1) Durant, J. L., Busby, W. F., Jr, Lafleur, A. L., Penman, B. W. and Crespi, C. L. (1996) Human cell mutagenicity of oxygenated, nitrated and unsubstituted polycyclic aromatic hydrocarbons associated with urban aerosols. Mutat. Res., 371, 123-157.

2) Schuetzle, D., Riley, T. L., Prater, T. J., Harvey, T. M. and Hunt, D. F. (1982) Analysis of nitrated polycyclic aromatic-hydrocarbons in diesel particulates. Anal. Chem., 54, 265-271.

3) Kameda, T., Inazu, K., Hisamatsu, Y., Takenaka, N. and Bandow, H. (2004) Determination of atmospheric nitro-polycyclic aromatic hydrocarbons and their precursors at a heavy traffic roadside and at a residential area in Osaka, Japan. Polycycl. Aromat. Compd., 24, 657-666.

4) Ball, L. M., Kohan, M. J., Inmon, J. P., Claxton, L. D. and Lewtas, J. (1984) Metabolism of 1 -nitro $\left[{ }^{14} \mathrm{C}\right]$ pyrene in vivo in the rat and mutagenicity of urinary metabolites. Carcinogenesis, $\mathbf{5}$, 1557-1564.

5) Howard, P. C., Beland, F. A. and Cerniglia, C. E. (1983) Reduction of the carcinogen 1-nitropyrene to 1 -aminopyrene by rat intestinal bacteria. Carcinogenesis, 4, 985-990.

6) Kameda, T., Akiyama, A., Toriba, A., Tang, N. and Hayakawa, K. (2011) Atmospheric formation of hydroxynitropyrenes from a photochemical reaction of particle-associated 1-nitropyrene. Environ. Sci. Technol., 45, 3325-3332.

7) Manabe, Y., Kinouchi, T. and Ohnishi, Y. (1985) Identification and quantification of highly mutagenic nitroacetoxypyrenes and nitrohydoroxypyrenes in diesel-exhaust particles. Mutat. Res., 158, 3-18.

8) Rosser, P. F., Ramachandran, P., Sangaiah, R., Austin, R. N., Gold, A. and Ball, L. M. (1996) Role of O-acetyltransferase in activation of oxidised metabolites of the genotoxic environmental pollutant 1-nitropyrene. Mutat. Res., 369, 209-220.
9) Ball, L. M., Kohan, M. J., Claxton, L. D. and Lewtas, J. (1984) Mutagenicity of derivatives and metabolites of 1-nitropyrene: activation by rat liver S9 and bacterial enzymes. Mutat. Res., 138, 113125.

10) El-Bayoumy, K. and Hecht, S. S. (1983) Identification and mutagenicity of metabolites of 1nitropyrene formed by rat liver. Cancer Res., 43, 3132-3137.

11) Fertuck, K. C., Matthews, J. B. and Zacharewski, T. R. (2001) Hydroxylated benzo[a]pyrene metabolites are responsible for in vitro estrogen receptor-mediated gene expression induced by benzo $[a]$ pyrene, but do not elicit uterotrophic effects in vivo. Toxicol. Sci., 59, 231-240.

12) Nishihara, T., Nishikawa, J., Kanayama, T., Dakeyama, F., Saito, K., Imagawa, M., Takatori, S., Kitagawa, Y., Hori, S. and Utsumi, H. (2000) Estrogenic activities of 517 chemicals by yeast twohybrid assay. J. Health Sci., 46, 282-298.

13) Vinggaard, A. M., Hnida, C. and Larsen, J. C. (2000) Environmental polycyclic aromatic hydrocarbons affect androgen receptor activation in vitro. Toxicology, 145, 173-183.

14) Kameda, T., Akiyama, A., Toriba, A., Tachikawa, C., Yoshita, M., Tang, N. and Hayakawa, K. (2008) Evaluation of endocrine disrupting activities of monohydroxylated derivatives of 1-nitropyrene by yeast two-hybrid assay. J. Health Sci., 54, 118122.

15) Lemaire, M., Guy, A., Roussel, J. and Guette, N. (1987) Nitrocyclohexadienones: A new class of nitrating agents. Tetrahedron, 43, 835-844.

16) Zielinska, B. (1990) A Facile Synthesis of 1Hydroxy-2-Nitropyrene and Its Applicability to Other Ortho-Substituted Hydroxynitroarenes. Polycycl. Aromat. Compd., 1, 207-211.

17) Maron, D. M. and Ames, B. N. (1983) Revised methods for the Salmonella mutagenicity test. $\mathrm{Mu}$ tat. Res., 113, 173-215.

18) Yahagi, T., Nagano, M. and Seino, Y. (1977) Mutagenicities of N-nitrosoamines on Salmonella. Mutat. Res., 48, 121-130.

19) Hirose, T., Morito, K., Kizu, R., Toriba, A., Hayakawa, K., Ogawa, S., Inoue, S., Muramatsu, M. and Masamune, Y. (2001) Estrogenic/antiestrogenic activities of benzo $[a]$ pyrene monohydroxy derivatives. J. Health Sci., 47, 552-558.

20) Hayakawa, K., Onoda, Y., Tachikawa, C., Hosoi, S., Yoshita, M., Chung, S. W., Kizu, R., Toriba, A., Kameda, T. and Tang, N. (2007) Estrogenic/antiestrogenic activities of polycyclic aromatic hydrocarbons and their monohydroxylated 
derivatives by yeast two-hybrid assay. J. Health Sci., 53, 562-570.

21) Löfroth, G., Nilsson, L., Arurell, E. and Yasuhara, A. (1984) Salmonella/microsome mutagenicity of 1-nitropyrene-2-ol, a nitropyrene phenol formed in the photolysis of 1-nitropyrene. Z. Naturforsch. C, 39, 193-195. 